\title{
Zoonotic Visceral Leishmania: Modeling and Control
}

Zamir Muhammad ${ }^{1 *}$ and Rahmat Ali $^{2}$

${ }^{1}$ Department of Mathematics, University of Science and Technology Bannu, Khyber Pakhtunkhwa, Pakistan

${ }^{2}$ Department of Mathematics, University of Malakand Chakdara Lower Dir, Khyber Pakhtunkhwa, Pakistan

\begin{abstract}
In this work we focus on the transmission dynamics of Visceral strains of leishmania, using mathematical model with two latent compartments in human. From the governing differential equations of the model, we find the reproductive number R0; the number of secondary infection and its biological interpretation. Using Routh- Hurwitz criteria on upper bound matrix, the threshold condition, for stability of the Disease Free State, is calculated. Finally we show that the disease free equilibrium is globally asymptotically stable if $R_{0}<<\xi 11$.
\end{abstract}

Keywords: Leishmaniasis; Basic reproductive number; Mathematical model; Local and global stability

\section{Introduction}

Visceral leishmaniasis is a vector-borne disease of humans and other mammals. This disease is caused by parasites of the Leishmania donovani complex. There are two main forms of visceral leishmania: (1) zoonotic visceral leishmaniasis (ZVL), which affects mainly young children and the domestic dog as its principal reservoir and (2) anthroponotic visceral leishmaniasis (AVL), this affects people of all ages, and infectious sand y transmit it from human to human via biting [1]. Visceral leishmaniasis (Vl) is severe and fatal. The average incubation period is 2-6 months; however it may vary from 10 days to one year [2,3]. Some of the patients recovered from V 1 , develops Post kala-Azar dermal leishmania with in the interval of 6 months to 3 years [4]. The vector latent period is assumed roughly to be 3 to 7 days $[5,6]$.

No doubt leishmania control is challenging because the control of both sandflies and the reservoir is di cult. The failure rate of treatment is high due the two factors. Clinical structure of disease, the response of human immune system and the drug resistance acquired by the species [7].

Motivated from Hashim et al. [8] and Shillor et al. [9], the authors did not consider Homogenous population. We in our work have considered the homogenous mixing of the population. The Reproductive number so calculated, depends upon the densities of humans, reservoirs and vectors, which highlights the importance of homogenous mixing. Also we have applied new concept for calculating threshold condition, for disease free state as developed by Kamgang and Sallet [10].

In this paper, we present a mathematical model for the transmission dynamic of leishmaniasis. The model of 10 compartments includes 2 exposed classes of human infected with visceral leishmaniasis and PKDL. These exposed classes were not considered previously in the models. We find positive invariant region and use next generation matrix method to find the basic reproduction number $R_{0}$. Using upper bound matrix $\mathrm{A}_{\mathrm{T}}(\mathrm{X})$ of the matrix $\mathrm{A}_{\mathrm{I}}(\mathrm{X})$, of the infected classes, the threshold number is found. Comparing $R_{0}$ and we find three values for $\mathrm{R}_{0}$. On the basis of these values, we discuss the dynamical behavior of the model. Finally we show the global stability of the disease free equilibrium, and the existence of endemic equilibrium.

\section{Model Formulation}

In this section we present the formulation of the model.
We divide the compartmental model of human, reservoir and vector populations into different classes. The human population consist of sub-classes, $\mathrm{S}_{h} ; \mathrm{E}_{1} ; \mathrm{I}_{1} ; \mathrm{P}_{2} ; \mathrm{R}_{1} ; \mathrm{E}_{12}$. Here $\mathrm{S}_{\mathrm{h}}$ represent the class of susceptible human, $\mathrm{E}_{1}$ is the $\mathrm{Vl}$ infected class, $\mathrm{E}_{12}$ is the class recovered from $\mathrm{Vl}$ and exposed to PKDL. $\mathrm{P}_{2}$ is the human class with PKDL and $\mathrm{R}_{1}$ is the human recovered class, $\mathrm{I}_{1}$ is the human class infectious with $\mathrm{Vl}$,

The total human population $\mathrm{N}_{\mathrm{h}}$ is

$$
\mathrm{N}_{\mathrm{h}}=\mathrm{S}_{\mathrm{h}}+\mathrm{E}_{1}+\mathrm{I}_{1}+\mathrm{E}_{12}+\mathrm{P}_{2}+\mathrm{R}_{1}
$$

The vector population is divided into two sub-classes $\mathrm{S}_{\mathrm{v}}(\mathrm{t})$ and $\mathrm{I}_{\mathrm{v}}(\mathrm{t})$, also the reservoir class is divided into $S_{r}(t)$ and $I_{r}(t)$.

$$
\mathrm{N}_{\mathrm{v}}(\mathrm{t})=\mathrm{S}_{\mathrm{v}}(\mathrm{t})+\mathrm{I}_{\mathrm{v}}(\mathrm{t}) ; \mathrm{N}_{\mathrm{r}}(\mathrm{t})=\mathrm{S}_{\mathrm{r}}(\mathrm{t})+\mathrm{I}_{\mathrm{r}}(\mathrm{t}) \text { : }
$$

After susceptible person, being bitten by infectious vector, he/she can't transmit leishmania virus immediately. We call this person as infected (exposed). When a susceptible vector $S_{v}(t)$, bite the infectious person, the vector moves from susceptible compartment to the infectious compartment $I_{v}(t)[11]$.

The interaction of human, reservoir and vector population is represented in the flowchart as shown in Figure 1.

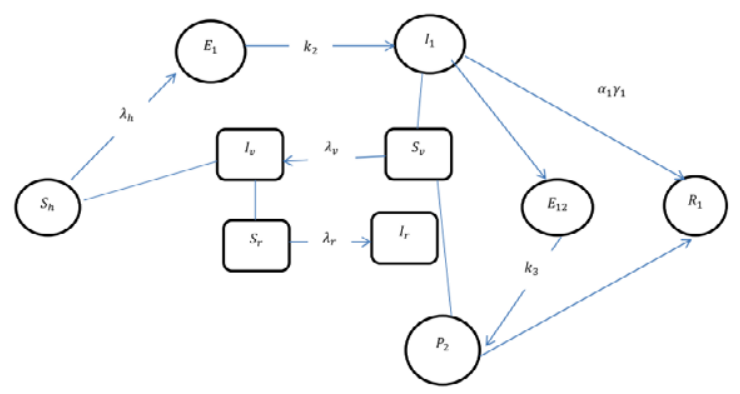

Figure 1: The interaction of human, reservoir and vector population.

*Corresponding author: Zamir M, Department of Mathematics, University of Science and Technology Bannu, Khyber Pakhtunkhwa, Pakistan, Tel: 0092331917093; E-mail: zamirburqi@yahoo.com

Received May 17, 2015; Accepted July 17, 2015; Published July 24, 2015

Citation: Muhammad Z, Ali R (2015) Zoonotic Visceral Leishmania: Modeling and Control. J Appl Computat Math 4: 238. doi:10.4172/2168-9679.1000238

Copyright: $\odot 2015$ Muhammad Z, et al. This is an open-access article distributed under the terms of the Creative Commons Attribution License, which permits unrestricted use, distribution, and reproduction in any medium, provided the original author and source are credited. 
Citation: Muhammad Z, Ali R (2015) Zoonotic Visceral Leishmania: Modeling and Control. J Appl Computat Math 4: 238. doi:10.4172/21689679.1000238

Page 2 of 6

The dynamical system for human, reservoir and vector population is given by

$$
\left\{\begin{array}{l}
\dot{S}=\Gamma_{h}-\left(\lambda_{h}+\mu_{h}\right) S_{h} \\
\dot{E}_{1}=\lambda_{h} S_{h}-\left(k_{2}+\mu_{h}\right) E_{1} \\
\dot{I_{1}}=k_{2} E_{1}-\left(\gamma_{1}+\delta_{1}+\mu_{h}\right) I_{1} \\
\dot{E_{12}}=\left(1-\alpha_{1}\right) \gamma_{1} I_{1}-\left(k_{3}+\mu_{h}\right) E_{12} \\
\dot{P_{2}}=k_{3} E_{12}-\left(\gamma_{2}+\beta_{1}+\delta_{2}+\mu_{h}\right) P_{2} \\
\dot{R}=\alpha_{1} \gamma_{1} I_{1}+\left(\gamma_{2}+\beta_{1}\right) P_{2}-\left(\mu_{h}\right) R \\
\dot{S_{r}}=\Gamma_{r}-\lambda_{r} S_{r}-\mu_{r} S_{r} \\
\dot{I}_{r}=\lambda_{r} S_{r}-\mu_{r} I_{r} \\
\dot{S_{v}}=\Gamma_{v}-\lambda_{v} S_{v}-\mu_{v} S_{v} \\
\dot{I}_{v}=\lambda_{v} S_{v}-\mu_{v} I_{v} .
\end{array}\right.
$$

The description of the parameters is given in Table 1.

The terms of interaction $\lambda_{\mathrm{h}}, \lambda_{\mathrm{r}}$ and $\lambda_{\mathrm{v}}$ are as under $\lambda_{h}=a b_{2} \frac{I_{v}}{N_{h}+N_{r}}$ is the average rate of infection rate of human with $\mathrm{Vl}$, from infectious sandfly.

$\lambda_{\mathrm{r}}$ is the average rate of infection of susceptible reservoir by infected sandfly.

$$
\lambda_{r}=a b \frac{I_{v}}{N_{h}+N_{r}} ; \mathrm{b} \text { is transmission probability of } \mathrm{V} 1 \text { to reservoir }
$$
from sandfly.

$\lambda_{v}=\frac{a}{N_{h}+N_{r}}\left(c_{2}\left(I_{1}+P_{2}\right)+c I_{r}\right)$, is the average rate of infection of sandfly with $\mathrm{Vl}$ strain from human or reservoir. Where $\mathrm{c}_{2}$ is the transmission probability of $\mathrm{Vl}$ from human in stage $\mathrm{I}_{1}$ and $\mathrm{P}_{2}$ to sandfly

\section{Mathematical Analysis of the Model}

In this section, we discuss invariant region, the disease free equilibrium point and reproductive number $\mathrm{R}_{0}$, of the system (1).

\section{Invariant region}

We have assumed all the parameters as nonnegative. Since the model is concerned with living population, therefore the state variables are assumed to be nonnegative at $\mathrm{t}=0$. The dynamic of overall population is given by the following differential equations.

$$
\begin{aligned}
& \dot{N}_{h}=\Gamma_{h}-\mu_{h} N_{h}-\delta_{1} I_{1}-\delta_{2} P_{2} \\
& \dot{N}_{r}=\Gamma_{r}-\mu_{r} N_{r}, \\
& \dot{N}_{v}=\Gamma_{v}-\mu_{v} N_{v} .
\end{aligned}
$$

If the human population is disease free, i.e. $\mathrm{I}_{1}=\mathrm{P}_{2}=0$, then equation (2) reduces to the form;

$$
\dot{N}_{h}=\Gamma_{h}-\mu_{h} N_{h} .
$$

Equilibrium in this case is

$N_{h_{u}}=\frac{\Gamma_{h}}{\mu_{h}}$.

From equation (2) and the fact that $\left(\delta_{1}+\delta_{2}\right) N_{h} \geq \delta_{1} I_{1}+\delta_{2}\left(p_{2}\right)$, we have

$\Gamma_{h}-\mu_{h} N_{h}-\left(\delta_{1}+\delta_{2}\right) N_{h} \leq \dot{N}_{h} \leq \Gamma_{h}-\mu_{h} N_{h}$

The lower bond for equation (7) is given by

$\dot{N}_{h}=\Gamma_{h}-\mu_{h} N_{h}-\left(\delta_{1}+\delta_{2}\right) N_{h}$.

\begin{tabular}{|c|c|c|c|}
\hline Notation & & Value & Resource \\
\hline $\mathrm{c}_{2}$ & Progression rate of VL in sand y(from human) & 0.22 & [14] \\
\hline a & Sandflies biting rate & 0.2856 day $^{-1}$ & [14] \\
\hline$\Gamma_{h}$ & Recruitment rate of human & 0.0015875 day $^{-1}$ & {$[15]$} \\
\hline$\Gamma_{v}$ & Recruitment rate of sandfly & 0.299 day $^{-1}$ & {$[16]$} \\
\hline$\Gamma_{r}$ & Recruitment rate of reservoir & 0.073 day $^{-1}$ & Assumed \\
\hline$\Gamma_{h}$ & Natural mortality rate of human & 0.00004 day $^{-1}$ & {$[16]$} \\
\hline$\mu_{v}$ & Natural mortality rate of Sandflies & 0.189 day $^{-1}$ & {$[16]$} \\
\hline$\mu_{r}$ & Natural mortality rate of Reservoirs & 0.000274 day $^{-1}$ & Assumed \\
\hline$\gamma_{2}$ & PKDL recovery rate after treatment & 0.033 day $^{-1}$ & {$[17]$} \\
\hline $1-\alpha_{1}$ & Developing PKDL rate after treatment & 0.36 day $^{-1}$ & {$[17]$} \\
\hline$\beta_{1}$ & PKDL natural healing rate & 0.00556 day $^{-1}$ & [17] \\
\hline c & Progression rate of VI in sandfly (from reseroir) & Variable & Variable \\
\hline $\mathrm{b}$ & Progression rate of $\mathrm{VI}$ in reservoir(from sandfly) & Variable & Variable \\
\hline$\gamma_{1}$ & Treatment rate of $\mathrm{VL}$ & variable & Assumed \\
\hline$\delta_{1}$ & VL induced death rate & 0.011 day $^{-1}$ & {$[18]$} \\
\hline $\mathrm{k}_{2}$ & $1 / \mathrm{k}_{2}$ is Incubation period of $\mathrm{vl}$ & 0.006555 day $^{-1}$ & {$[19]$} \\
\hline $\mathrm{k}_{3}$ & $1 / \mathrm{k}_{3}$ is Incubation period of $\mathrm{PKDL}$ & 0.004925925 day $^{-1}$ & {$[2,20]$} \\
\hline$\delta_{2}$ & PKDL induced death rate & assumed & Assumed \\
\hline$b_{2}$ & Progression rate of VL in human (from sandfly) & 0.0714 day $^{-1}$ & [21] \\
\hline
\end{tabular}

The equilibrium of equation (8) is

$N_{h_{l}}=\frac{\Gamma_{h}}{\mu_{h}+\delta_{1}+\delta_{2}}$.

With the initial condition

Table 1: Description of the parameters. 


$$
\mathrm{N}_{\mathrm{h}}(0)=\mathrm{N}_{0} \text {. }
$$

If $\mathrm{N}_{\mathrm{u}}$, and $\mathrm{N}_{\mathrm{l}}$, denote the solution of equation (5) and equation (8), then any solution of equation (2), satisfy

$$
\mathrm{N}_{1} \leq \mathrm{N}_{\mathrm{h}} \leq \mathrm{N}_{\mathrm{u}} \text {. }
$$

Consider the biological feasible region $\Omega$ given by:

$$
\Omega=\left[\left(S_{h}, E_{1}, I_{1}, P_{2}, R_{1}, E_{12}, S_{r}, I_{r}, S_{v}, I_{v}\right) \in R_{+}^{10}, N_{h} \leq \frac{\Gamma_{h}}{\mu_{h}} ; N_{r} \leq \frac{\Gamma_{r}}{\mu_{r}} ; N_{v} \leq \frac{\Gamma_{v}}{\mu_{v}}\right] .
$$

From equation (2), using standard comparison theorem, we have

$$
N_{h} \leq N_{h}(0) e^{-\mu(t)}+\frac{\Gamma_{h}}{\mu_{h}}\left(1-e^{-\mu_{h}(t)}\right) \text {. }
$$

So

$$
N_{h} \rightarrow \frac{\Gamma_{h}}{\mu_{h}} \text { as } t \rightarrow \infty .
$$

Similarly

$$
\left[N_{v} \rightarrow \frac{\Gamma_{v}}{\mu_{v}} \text { and } N_{r} \rightarrow \frac{\Gamma_{r}}{\mu_{r}}\right] \text { and } t \rightarrow \infty
$$

Hence is positively invariant domain, and the model is epidemiologically and mathematically well posed.

Let us de ne a new region $G$ as

$$
G=\left\{X \in \Omega ; N_{h_{l}} \leq N_{h} \leq N_{h_{u}} ; N_{r} \leq \frac{\Gamma_{r}}{\mu_{r}} ; N_{v} \leq \frac{\Gamma_{v}}{\mu_{v}}\right\} .
$$

where

$$
X=\left(S_{h}, E_{1}, I_{1}, E_{12}, P_{2}, R_{1}, S_{r}, I_{r}, S_{v}, I_{v}\right)^{T} .
$$

Clearly $\mathrm{G}$ is the sub region of $\Omega$. In light of equation (3), equation (4) and equation (11), it is reasonable to work on $G$ instead of $\Omega$.

\section{Disease free equilibrium}

The disease free equilibrium of the model (1) is given by:

$$
X_{0}=\left(\frac{\Gamma_{h}}{\mu_{h}}, 0,0,0,0,0, \frac{\Gamma_{r}}{\mu_{r}}, 0\right),
$$

\section{Reproductive number}

The number of secondary infections occurring in completely susceptible population by introducing an infectious individual to the population is called reproductive number $\mathrm{R}_{0}[12]$. In order to find the basic reproductive number, we use next generation method for $\mathrm{R}_{0}=$ $\left(-\mathrm{FV}^{-1}\right),[13]$. Where is spectral radius? And

$$
F=\left(\begin{array}{cccccc}
0 & 0 & 0 & 0 & 0 & m_{1} \\
0 & 0 & 0 & 0 & 0 & 0 \\
0 & 0 & 0 & 0 & 0 & 0 \\
0 & 0 & 0 & 0 & 0 & 0 \\
0 & 0 & 0 & 0 & 0 & m_{2} \\
0 & m_{3} & 0 & m_{4} & m_{5} & 0
\end{array}\right), V=\left(\begin{array}{cccccc}
-a_{1} & 0 & 0 & 0 & 0 & 0 \\
k_{2} & -a_{2} & 0 & 0 & 0 & 0 \\
0 & d_{2} & -a_{3} & 0 & 0 & 0 \\
0 & 0 & k_{3} & -a_{4} & 0 & 0 \\
0 & 0 & 0 & 0 & -\mu_{r} & 0 \\
0 & 0 & 0 & 0 & 0 & -\mu_{v}
\end{array}\right),
$$

Here

$$
\begin{aligned}
& m_{1}=a b_{2} \frac{\Gamma_{h} \mu_{r}}{\mu_{r} \Gamma_{h}+\mu_{h} \Gamma_{r}}, m_{2}=a b \frac{\Gamma_{r} \mu_{h}}{\mu_{r} \Gamma_{h}+\mu_{h} \Gamma_{r}}, \\
& m_{3}=a c_{2} \frac{\mu_{r} \mu_{h} \Gamma_{v}}{\mu_{v}\left(\mu_{r} \Gamma_{h}+\mu_{h} \Gamma_{r}\right)}, \quad m_{4}=a c_{2} \frac{\mu_{r} \mu_{h} \Gamma_{v}}{\mu_{v}\left(\mu_{r} \Gamma_{h}+\mu_{h} \Gamma_{r}\right)}, m_{5}=a c \frac{\mu_{r} \mu_{h} \Gamma_{v}}{\mu_{v}\left(\mu_{r} \Gamma_{h}+\mu_{h} \Gamma_{r}\right)} .
\end{aligned}
$$

with

$$
a_{1}=k_{2}+\mu_{h}, \quad a_{2}=\gamma_{1}+\delta_{1}+\mu_{h} \quad a_{3}=k_{3}+\mu_{h} a_{4}=\gamma_{2}+\beta_{1}+\delta_{2}+\mu_{h}
$$

$$
d_{2}=\left(1-\alpha_{1}\right) \gamma_{1} \text {. }
$$

After simplification, we get reproduction number

$$
R_{0}=\left[\frac{m_{2} m_{5}}{\mu_{r} \mu_{v}}+\frac{k_{2} m_{1} m_{3}}{a_{1} a_{2} \mu_{v}}+\frac{d_{2} k_{3} k_{2} m_{4} m_{1}}{\mu_{v} a_{1} a_{2} a_{3} a_{4}}\right]^{\frac{1}{2}} .
$$

We can further simplify to get $R_{0}=\sqrt{R_{a}+R_{b}}$ where $R_{a}=R_{1} R_{2}, R_{b}=R_{3} R_{4}$.

$$
\begin{aligned}
& R_{1}=\frac{a b \mu_{h} \Gamma_{v}}{\mu_{v}\left(\Gamma_{h} \mu_{r}+\Gamma_{r} \mu_{h}\right)}, R_{2}=\frac{a c \mu_{h} \Gamma_{r}}{\mu_{v}\left(\Gamma_{h} \mu_{r}+\Gamma_{r} \mu_{h}\right)}, \\
& R_{3}=\frac{a b_{2} \mu_{r} \Gamma_{h}}{\mu_{v}\left(\Gamma_{h} \mu_{r}+\Gamma_{r} \mu_{h}\right)}, R_{4}=\frac{a c_{2} \mu_{r} \mu_{h} \Gamma_{v}}{\mu_{v}\left(\Gamma_{h} \mu_{r}+\Gamma_{r} \mu_{h}\right)}\left[\frac{k_{2}}{a_{1} a_{2}}\left(1+\frac{d_{2} k_{3}}{a_{3} a_{4}}\right)\right] .
\end{aligned}
$$

The term R1 indicates that if sandfly is infectious and the reservoir is susceptible, the contact would result the transmission of $\mathrm{Vl}$ from sand $y$ to reservoir. The term $R_{2}$ indicates the transmission of $V_{1}$ from reservoir to sand $y$. So the term $R_{a}$ indicate the transmission of $V_{1}$ between sandfly and reservoir. Similarly the term $R_{b}$ indicates the transmission of $\mathrm{Vl}$ between human and sand fly. The term $\mathrm{R}_{\mathrm{a}}$ and $\mathrm{R}_{\mathrm{b}}$ both denote the transmission of visceral strains of leishmania. There is no term representing the transmission of PKDL because it is the silent complication of V $\mathrm{l}$. When a susceptible vector bites human/reservoir infected with PKDL, the vector does not transmit PKDL but transmit $\mathrm{V}_{1}$ to the next victim. So $\mathrm{R}_{0}$ is biologically sensible.

\section{Stability Analysis}

In this section, we discuss the relation between additional threshold number and basic $\xi$ reproductive number $\mathrm{R}_{0}$, to find the global stability of the disease free equilibrium, and existence of endemic equilibrium of the system (1).

Proposition: The disease free equilibrium is locally asymptotically stable if $\mathrm{R}_{0}<1$ and unstable if $\mathrm{R}_{0}>1$.

Proof: For the proof of this result verify the reference [13].

\section{Global stability of the disease free equilibrium}

To find the global stability of the disease free equilibrium of the system (1), we state some definitions $[9,10]$.

Definition 1: An $\mathrm{m} \times \mathrm{m}$ matrix, for $\mathrm{m}>2$ is called irreducible if for any proper sub-set $\mathrm{I}$ of $\{1,2, \ldots, \mathrm{m}\}, \exists, p \in I$ and $q \notin I$ such that $A_{(p, q)} \neq 0$

Definition 2: The matrix M is said to be Metzler matrix if $A_{(p, q)} \geq 0$ for $p \neq q$.

Definition 3: The compact set $M \subset \Omega$ is called stable for the dynamical system defined on $\Omega$ if for every trajectory initiated from a point in $U$ is in $W$, for all $t \geq 0$. Here $U$ and $W$ are neighborhoods of $M$.

Definition 4: A compact set $N \subset D$ is called an attractor for a dynamical system defined on $\mathrm{D}$ if there exist a nbhds $\mathrm{X}$ and $\mathrm{Y}$ of $\mathrm{N}$ such that for every point $X \in x$, there exists a time $t_{x y}>0$, such that every trajectory initiated at $\mathrm{x}$, belongs to $\mathrm{Y}$ for $\mathrm{t}>\mathrm{t}_{\mathrm{x}, \mathrm{y}}$. The largest set $\mathrm{X}$ is called a bassin of attraction.

If $\mathrm{X}=\mathrm{D}$ the set $\mathrm{N}$ is then called global attractor. A set $\mathrm{N}$ which is both stable and a global attractor is called globally asymptotically stable.

Theorem: The set $G$ is globally asymptotically stable for the dynamical system (1) defined on $\Omega$. 


\section{Proof: Let}

$$
\begin{aligned}
& N_{r}(0)=N_{r}^{0}, \\
& N_{v}(0)=N_{v}^{0},
\end{aligned}
$$

be initial conditions associated with equation (3) and equation (4). And for $\epsilon>0, B_{\epsilon}(G)$ be defined as;

$$
\begin{aligned}
& B_{\epsilon}(G)=\left\{X \in \Omega ; N_{-} \epsilon<N_{h}<N_{h_{u}}+\epsilon ; N_{r}^{0}-\epsilon<N_{r}<N_{r_{u}}+\epsilon ; N_{v}^{0}-\epsilon<N_{v}<N_{v_{u}}+\epsilon\right\} \\
& \text { where } \\
& X=\left(S_{h}, E_{1}, I_{1}, E_{12}, P_{2}, R_{1}, S_{r}, I_{r}, S_{v}, I_{v}\right)
\end{aligned}
$$

Since the collection $\left\{B_{\epsilon}(G), \epsilon>0\right\}$ is a complete neighborhood system of the compact set G. So X and Y as discussed in above definitions, also belong to this collection.

Consider an arbitrary $\epsilon>0$. The points $N_{r_{u}}, N_{v_{u}}, N_{h_{l}}, N_{h_{u}}$ are globally asymptotically stable equilibria of the dynamical system defined by equation (3), equation (4), equation (5), and equation (8) on $(0, \infty)$.

Foranyinitialpointofthemodel $(1), N_{h}^{0} \in(0, \infty), N_{r}^{0} \in(0, \infty), N_{v}^{0} \in(0, \infty)$ Hence there exists $t_{\epsilon}>0$ so that for any $t>t_{\epsilon}$ we have

$$
\begin{aligned}
& N_{h_{l}}-\epsilon<N_{l} \leq N_{h} \leq N_{u}<N_{h_{u}}+\epsilon, \\
& N_{r_{l}}-\epsilon<N_{r}<N_{r_{u}} \epsilon, \\
& N_{v_{l}}-\epsilon<N_{v}<N_{v_{u}}+\epsilon, \\
& \Rightarrow X=\left(S_{h}, E_{1}, I_{1}, E_{12}, S_{r}, I_{r}, S_{v}, I_{v}\right) \in B_{\epsilon}(G) .
\end{aligned}
$$

Thus $\mathrm{G}$ is global attractor.

Next to show that $G$ is stable

On the basis of monotonicity of $N_{l}, N_{u}, N_{r}, N_{v}$, we have

$$
\left\{\begin{array} { l } 
{ N _ { h _ { l } } - \epsilon < N _ { h } ^ { 0 } < N _ { h _ { u } } + \epsilon } \\
{ N _ { r _ { l } } - \epsilon < N _ { r } ^ { 0 } < N _ { r _ { l } } + \epsilon } \\
{ N _ { v _ { l } } - \epsilon < N _ { v } ^ { 0 } < \hat { N } _ { v _ { l } } + \epsilon }
\end{array} \Rightarrow \left\{\begin{array}{l}
N_{h_{l}}-\epsilon<N_{l} \leq N_{h} \leq N_{u}<N_{h_{u}}+\epsilon \\
N_{r}^{*}-\epsilon<N_{r}<N_{r}^{*}+\epsilon \\
N_{v}^{*}-\epsilon<N_{v}<N_{v}^{*}+\epsilon .
\end{array}\right.\right.
$$

Thus we have shown that any solution of the model (1), starting from a point in $B_{\epsilon}(G)$, remains in $B_{\epsilon}(G)$. So $\mathrm{G}$ is stable. Thus $\mathrm{G}$ is globally asymptotically stable. Hence we can now study the system (1) on $\mathrm{G}$, instead of $\Omega$.

Theorem: Let a positive system be defined on set $\Omega \subseteq R^{n}$ and let $\varepsilon \subset \Omega$ be globally asymptotically stable. Let $\mathrm{M}$ be the largest invariant sub set of $\mathcal{E}$. Then $\mathrm{M}$ is globally asymptotically stable on $\Omega$. Particularly if $M=\left\{x^{*}\right\}$ where $x^{*}$ is equilibrium point of the system with basin of attraction containing $\varepsilon$. Then $x^{*}$ is GAS for the system on $\Omega$.

Proof: For the proof of the theorem verify the reference [9] theorem (5). To prove the global stability of the disease free equilibrium, we use theorem (4.3) of [10].

For this let

$$
X=\left(S_{h}, R_{1}, S_{v}, S_{r}, E_{1}, I_{1}, E_{12}, P_{2}, I_{r}, I_{v}\right)^{T} .
$$

Now for global asymptotic stability of the disease free equilibrium of the system(1) on smaller set G, we decompose X as, Xs and XI of noninfected and infected, humans reservoirs and sandies, such that

$$
X_{s}=\left(S_{h}, R_{1}, S_{r}, S_{v}\right)^{T} \text {, }
$$

$$
X_{I}=\left(E_{1}, I_{1}, E_{12}, P_{2}, I_{r}, I_{v}\right)^{T} .
$$

So the model can now be written as

$$
\dot{X}=A(X)+E_{X} \rightarrow\left\{\begin{array}{l}
X_{S}=A_{S}(X) X_{S}+E_{S} \\
X_{I}=A_{I}(X) X_{I}
\end{array}\right.
$$

where

$$
\begin{aligned}
& A_{S}=\left(\begin{array}{cccc}
-\left(\mu_{h}+\lambda_{h}\right) & 0 & 0 & 0 \\
0 & -\mu_{h} & 0 & 0 \\
0 & 0 & -\left(\mu_{r}+\lambda_{r}\right) & 0 \\
0 & 0 & 0 & -\left(\mu_{v}+\lambda_{v}\right)
\end{array}\right) \\
& E_{S}=\left(\Gamma_{h}, \gamma_{1} \alpha_{1} I_{1}+\left(\gamma_{2}+\beta_{1}\right) P_{2}, \Gamma_{r}, \Gamma_{v}\right)^{T} .
\end{aligned}
$$

And the matrix $A_{I}(X)$ is given by

$$
A_{I}(X)=\left(\begin{array}{cccccc}
-a_{1} & 0 & 0 & 0 & 0 & \frac{a b_{2} S_{h}}{N_{h}+N_{r}} \\
k_{2} & -a_{2} & 0 & 0 & 0 & 0 \\
0 & d_{2} & -a_{3} & 0 & 0 & 0 \\
0 & 0 & k_{3} & -a_{4} & 0 & 0 \\
0 & 0 & 0 & 0 & -\mu_{r} & \frac{a b S_{r}}{N_{h}+N_{r}} \\
0 & \frac{a c_{2} S_{v}}{N_{h}+N_{r}} & 0 & \frac{a c_{2} S_{v}}{N_{h}+N_{r}} & \frac{a c S_{r}}{N_{h}+N_{r}} & -\mu_{v}
\end{array}\right)
$$

We restrict the domain of the system (1) from $G$ to $G$, to ensure the irre-ducibility of $\mathrm{A}_{\mathrm{I}}(\mathrm{X})$, such that $\bar{G}=\left\{X ; X \in G, X_{S} \neq 0\right\}$.

The set $\bar{G}$ is positively invariant because only the initial point of any trajectory can have $\mathrm{X}_{\mathrm{s}}=0$, Putting $\dot{S}_{h}=\dot{R}_{1}=\dot{S}_{r}=\dot{S}_{v}=0$, in the system (1), we have $S_{h}>0, R_{1}>0, S_{r}>0, S_{v}>0$.

So all of the diagonal entries of $\mathrm{A}_{\mathrm{I}}(\mathrm{X})$ are nonnegative, hence $\mathrm{A}_{\mathrm{I}}(\mathrm{X})$ is metzler and irreducible $\forall X \in \bar{G}$. result

Since diagonal entries of $\mathrm{A}_{\mathrm{s}}$ are negative. So we state the following

Proposition: Let $X_{s}^{0}$ be the non-infected class of the total population, then

$$
X_{s}^{0}=\left(S_{h}^{0}, R_{1}^{0}, S_{r}^{0}, S_{v}^{0}\right)=\left(\frac{\Gamma_{h}}{\mu_{h}}, 0, \frac{\Gamma_{r}}{\mu_{r}}, \frac{\Gamma_{v}}{\mu_{v}}\right)
$$

is globally asymptotically stable equilibrium point of the system (1) reduced to the sub-domain $\left\{X \in \bar{G} ; X_{I}=0\right\}$.

Corollary: The system (14) is globally asymptotically stable if there exist a matrix $\bar{A}_{I}$ such that

$$
A_{I}(X) \leq \bar{A}_{I} X \in \bar{G} .
$$

and if

$$
\begin{aligned}
& A_{I}(\bar{X})=\bar{A}_{I} \text { for some } \bar{X}=\left(\bar{X}_{s}, \bar{X}_{I}\right) \text { then } \bar{X}_{I}=0, \\
& \alpha\left(\bar{A}_{I}\right) \leq 0
\end{aligned}
$$

Where $\alpha$ is stability modulus or the largest real part of the eigen values of $\bar{A}_{I}$ 


\section{Proof:}

Since

$$
\frac{1}{N_{h}+N_{r}} \leq \frac{1}{N_{h_{l}}+N_{r}}
$$

So the upper bond of $A_{I}(X)$ denoted by $\bar{A}_{I}$ is given by

$$
\overline{A_{I}}(X)=\left(\begin{array}{cccccc}
-a_{1} & 0 & 0 & 0 & 0 & \frac{a b_{2} S_{h}^{0}}{N_{h_{l}}+N_{r}} \\
k_{2} & -a_{2} & 0 & 0 & 0 & 0 \\
0 & 0 & d_{2} & -a_{3} & 0 & 0 \\
0 & 0 & -k_{3} & -a_{4} & 0 & 0 \\
0 & 0 & 0 & 0 & -\mu_{r} & \frac{a b_{2} S_{r}^{0}}{N_{h_{l}}+N_{r}} \\
0 & \frac{a c_{2} S_{v}^{0}}{N_{h_{l}}+N_{r}} & 0 & \frac{a c_{2} S_{v}^{0}}{N_{h_{l}}+N_{r}} & \frac{a c S_{v}^{0}}{N_{h_{l}}+N_{r}} & -\mu_{v}
\end{array}\right),
$$

and

Clearly $A_{I}(X) \leq \overline{A_{I}}(X)$ as $\frac{1}{N_{h}+N_{r}} \leq \frac{1}{N_{h_{l}}+N_{r}}$

And

$A_{I}(X)=\overline{A_{I}}(X)$ only if; $S_{h}=S_{h}^{0}, R_{1}=R_{1}^{0}, S_{r}=S_{r}^{0}, S_{v}=S_{v}^{0}$

Thus $\mathrm{H}_{4}$ of theorem (4.3) holds [10], equivalently equation (15) and equation (16), hold.

To show that $\mathrm{H}_{5}$ or equation (18) holds, we state the following theorem.

Theorem: The metzler matrix satisfy the axiom $H_{5} ; \alpha\left(\bar{A}_{I}\right) \leq 0$ if the basic reproductive number $\mathrm{R}_{0}$ satisfy the inequality; $R_{0} \leq \xi$, where $\xi$, is the additional threshold number given by

$$
\begin{aligned}
& \xi=\frac{a^{2} b_{2} c_{2} \mu_{r}\left(\mu_{h}+\delta_{1}+\delta_{2}\right)^{2} \Gamma_{v} \Gamma_{r}}{\mu_{h} \mu_{v}^{2}\left(\mu_{r} \Gamma_{h}+\left(\mu_{h}+\delta_{1}+\delta_{2}\right) \Gamma_{r}\right)^{2}}\left(\frac{k_{2}}{a_{2} a_{1}}\left(1+\frac{d_{2} k_{3}}{a_{2} a_{3}}\right)\right) \\
& +\frac{a^{2} b c\left(\mu_{h}+\delta_{1}+\delta_{2}\right)^{2} \Gamma_{v} \Gamma_{r}}{\mu_{v}^{2}\left(\mu_{r} \Gamma_{h}+\left(\mu_{h}+\delta_{1}+\delta_{2}\right) \Gamma_{r}\right)^{2}}
\end{aligned}
$$

Proof: We decompose the matrix $\overline{A_{I}}$ in the blocks such that

$$
\overline{A_{I}}=\left(\begin{array}{cc}
L & M \\
P & Q
\end{array}\right),
$$

where $\mathrm{L}, \mathrm{M}, \mathrm{P}, \mathrm{Q}$ are $3 \times 3$ sub-matrices. The matrix $\overline{A_{I}}$ is stable if $\mathrm{S}$ and $Q-P L^{-1} M$ are metzler stable. Here $\mathrm{S}$ is metzler stable, because all its off diagonal entries are nonnegative, and all the eigen values are negative.

Let

$Y=Q-P L^{-1} M$

Then $\overline{A_{I}}$ is stable if $\mathrm{Y}$ is stable.

And $\mathrm{Y}$ is stable if $\operatorname{det}(Y) \geq 0$

This means that $\alpha\left(\overline{A_{I}}\right) \leq 0$ only if

$\frac{n_{2} n_{5}}{\mu_{r} \mu_{v}}+\frac{k_{2} n_{1} n_{3}}{a_{1} a_{2} \mu_{v}}+\frac{d_{2} k_{2} k_{3} n_{1} n_{4}}{a_{1} a_{2} a_{3} a_{4} \mu_{v}}-1<0, \Rightarrow \frac{n_{2} n_{5}}{\mu_{r} \mu_{v}}+\frac{k_{2} n_{1} n_{3}}{a_{1} a_{2} \mu_{v}}+\frac{d_{2} k_{2} k_{3} n_{1} n_{4}}{a_{1} a_{2} a_{3} a_{4} \mu_{v}}<1$, where $n_{1}=\frac{a b_{2} S_{h}^{0}}{N_{h_{l}}+N_{r}}, n_{2}=\frac{a b S_{r}^{0}}{N_{h_{l}}+N_{r}}, n_{3}=\frac{a c_{2} S_{v}^{0}}{N_{h_{l}}+N_{r}}, n_{4}=\frac{a c_{2} S_{v}^{0}}{N_{h_{l}}+N_{r}} \quad n_{5}=\frac{a c S_{v}^{0}}{N_{h_{l}}+N_{r}}$,

At the disease free equilibrium,

$$
\begin{aligned}
& S_{r}=S_{r}^{0}=\frac{\Gamma_{r}}{\mu_{r}}, S_{h}=S_{h}^{0}=\frac{\Gamma_{h}}{\mu_{h}}, R_{1}=R_{1}^{0}=\frac{\Gamma_{h}}{\mu_{h}}, \\
& N_{h_{l}}+N_{r}=\frac{\Gamma_{h} \mu_{r}+\Gamma_{r}\left(\mu_{h}+\delta_{1}+\delta_{2}\right)}{\mu_{r}\left(\mu_{h}+\delta_{1}+\delta_{2}+\delta_{3}\right)} .
\end{aligned}
$$

By putting these values in above equation, we have

$$
\frac{a^{2} b c\left(\mu_{h}+\delta_{1}+\delta_{2}\right)^{2} \Gamma_{v} \Gamma_{r}}{\mu_{v}^{2}\left(\Gamma_{h} \mu_{r}+\Gamma_{r} \mu_{h}+\delta_{1}+\delta_{2}\right)^{2}}+\frac{a^{2} b_{2} c_{2}\left(\mu_{h}+\delta_{1}+\delta_{2}\right) \mu_{r}^{2} \Gamma_{h} \Gamma_{v}}{\mu_{v}^{2}\left(\Gamma_{h} \mu_{r}+\Gamma_{r} \mu_{h}+\delta_{1}+\delta_{2}\right)^{2}}\left[\frac{k_{2}}{a_{2} a_{2}}\left(1+\frac{d_{2} k_{3}}{a_{3} a_{4}}\right)\right] \geq 1
$$

We take this value as $\xi$. Thus $\mathrm{H}_{5}$ or equation(17) holds, if $\xi \geq 1$. Also $R_{0}<\xi$. So using theorem (4.3) of [10], we claim the following result.

Theorem: If the parameters of the model satisfy the condition $\alpha\left(\overline{A_{I}}\right) \leq 0$, then the disease free equilibrium of the system (1) is globally asymptotically stable.

\section{Simulation results of the model}

In the Figure 2 below, we have reduced the treatment rate of both $\mathrm{Vl}$ infected and PKDL infected humans, in the sense that we have used drugs other than sodium stibogluconate (expensive medicine) or that the hospital is far away or that the case is not properly diagnosed leading to wrong treatment. No mass awareness program is lunched for vector control. Taking $\gamma_{1}=\gamma_{2}=0: 023, a=0: 2856$ (normal); and $\alpha_{1}=0: 064$.
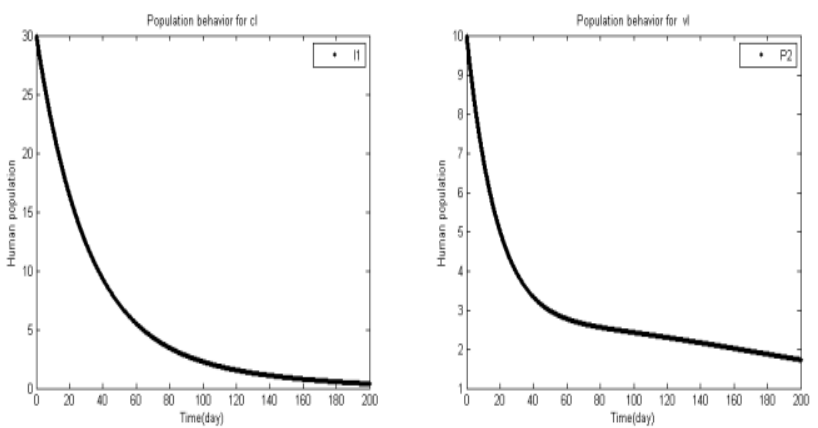

Figure 2: Population behavior graph 1
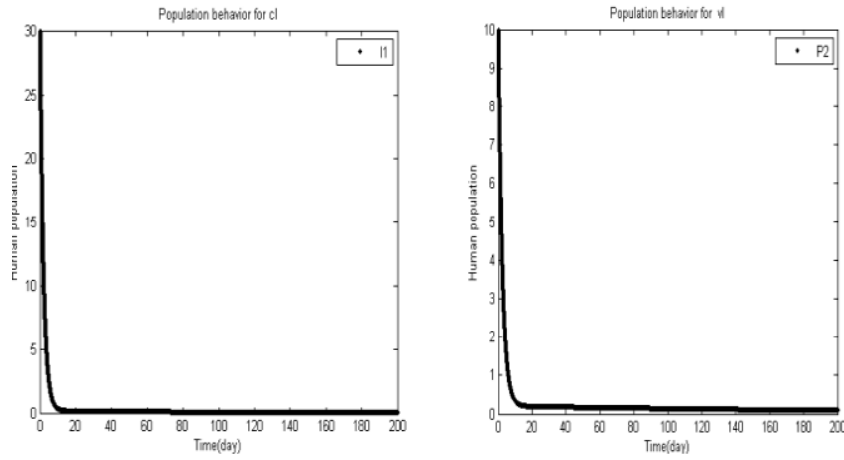

Figure 3: Population behavior graph 2. 
The graph shows that it takes long time to eradicate the diseases.

In Figure 3 we have increased the treatment rate for both $\mathrm{Vl}$ and PKDL and also a proper arrangement for vector control. Taking $\gamma_{1}=0: 5$, $\gamma_{2}=0: 4$, biting of sandfly a $=0.1856$ medicine effectiveness $\alpha_{1}=0: 74$. The graph shows that with in short time the disease can be eradicated.

\section{Conclusion}

In this work a mathematical model of leishmania transmission was presented. The novelty of the model is, the homogenous mixing of human, reservoir and vector. The basic reproduction number $\mathrm{R}_{0}$ so calculated, depends upon the density of human, vectors and reservoirs, which highlights the importance of homogenous mixing. R0 is most sensitive to $\mathrm{a} ; \mathrm{b}$ and $\mathrm{c}$ and can have value greater than 1 (endemic state), if $a$; sand y biting rate, $b$; transmission probability of either strain in reservoir from sand $y$ and $c$, transmission probability of either strain in sand y from reservoir, were not controlled. For this, different measures to control phlebotomine sandfies, like residual spraying of dwellings and animal shelters, insecticide treated nets; application of repellents/ insecticides to skin or to fabrics and impregnated dog collars may be taken. Sand y is susceptible to all the major insecticidal groups. In ZVL foci, where dogs are the unique domestic reservoir, a reduction in Leishmania transmission would be expected if we could combine an effective mass treatment of infected dogs with a protection of both healthy and infected dogs from the sand y bites. Since sand y can y up to the range of $1 \mathrm{~km}$, so leishmania transmission in dogs can be controlled, if they were kept away at least by $1 \mathrm{~km}$, from villages and cities. The disease can be controlled in human within a short time, however in reservoir class; the disease control takes long time. It is suggested to cull PCR+ dogs; this strategy gives imminent results in disease control.

\section{References}

1. Molina R, Gradoni L, Alvar J (2003) Hiv and the transmission of leishmania Ann Trop Med Parasitol 97: 29-45.

2. Badaro R, Jones TC, Carvalho EM, Sampaio D, Reed SG (1986) New perspectives on a subclinical form of visceral leishmaniasis. J Infect Dis 15 1003-1011.

3. WHO (1996) Manual of Visceral Leishmaniasis Control.

4. Lowth M (2014) Leishmaniasis. Patient co, UK.

5. Sacks DL, Perkins PV (1985) Development of infective stage leishmania promastigotes within phlebotomine sandflies. Am J Trop Med Hyg 34: 456 467.

6. Sacks DL, Perkins PV (1984) Identification of an infective stage of leish-mania promastigotes. Science 223: 1417-1419.
7. Porrozzi R, Teva A, Amaral VF, Santos MV, Grimaldi GJR (2004) Crossimmunity experiments between different species or strains of leish-mania in rhesus macaques (macaca mulatta). Am J Trop Med Hyg 17: 297-305.

8. Elmojtaba IM, Mugisha JYT, Hashim MHA (2010) Modelling the role of crossimmunity between two di erent strains of leishmania. Nonlinear Analysis: Real World Applications 11: 2175-2189.

9. Dumont AY, Lumba JMS, Shillor M (2013) Stability analysis and dynamics preserving nonstandard finite difference scheme for a malaria model, Mathematical population studies. International Journal of Mathematical Demography 20: 101-122.

10. Kamgang JC, Sallet G (2008) Computation of threshold conditions for epidemiological models and global stability of the disease-free equilibrium. Mathematical Biosciences 21: 31-12.

11. Zaman g, Lashari AA, Chohan MI (2012) Dynamical features of dengue with saturating incidence rate. International Journal of pure and Applied Mathmatics 76: $483-402$.

12. Diekmann O, Heesterbeek JAP, Metz J (1990) On the definition and computation of the basic reproduction ratio $\mathrm{R} 0$ in models for infectious diseases in heterogeneous populations. J Math Biol 28: 365-382.

13. Driessche $P$, Watmough $J$ (2002) Reproduction numbers and the sub-treshold endemic equilibria for compartmental models of disease trans-mission. Math Biosci 180: 29-48.

14. Fialho RF, Schall JJ (1995) Thermal Ecology of Malarial parasite and its insect vector: Consequence for parasites transmission success. Journal of Animal Ecology 46(5): 553-562.

15. Gemperli, Vounatsou P, Sogoba N, Smith T (2006) Malaria mapping using transmission models: Application to survey data. Amer J Epidemiol 163: 289297.

16. Kasap OE, Alten B (2006) Comparative demography of the sandy Phlebotomus papatasi (Diptera: Psychodidae) at constant temperatures. J Vector Ecol 31(2): $378-385$

17. Gasim G, El Hassan AM, Kharazmi A, Khalil EAG, Ismail A, et al. (2000) The development of post kala-azar dermal leishmaniasis (PKDL) is associated with acquisition of Leishmania reactivity by peripheral blood mononuclear cells (PBMC). Clin Exp Immunol 119: 523-529.

18. Sundar S, Agrawal G, Rai M, Makharia MK, Murray HW (2001) Treatment of Indian visceral leishmaniasis with single or daily infusion of low dose liposomal amphotericin B: Randomised trial. BMJ 323: 419-422.

19. Chappuis F, Sunder S, Hailu A, Ghalib H, Rigal S, et al (2007) Visceral leishmania diagnosis,treatment and control. Nature Reviews 5: 873-882.

20. Zijltra E, Musa A, Khalil E, Elhassan I, Elhassan A (2003) Post-Kala-azar dermal leishmaniasis. The Lancet infectious diseases 3(2): 87-98.

21. Lainson R, Ryan L, Shaw JJ (1987) Infective stages of Leishmania in the sand y vector and some observations on the mechanism of transmission. Mem Inst Oswaldo Cruz 82(3): 421-424. 